\title{
BMJ Open Collaborative care for comorbid depression and diabetes: a systematic review and meta-analysis
}

\author{
Evan Atlantis, ${ }^{1,2}$ Paul Fahey, ${ }^{3}$ Jann Foster ${ }^{1}$
}

To cite: Atlantis E, Fahey $P$, Foster J. Collaborative care for comorbid depression and diabetes: a systematic review and meta-analysis. BMJ Open 2014:4:e004706. doi:10.1136/bmjopen-2013004706

- Prepublication history and additional material is available. To view please visit the journal (http://dx.doi.org/ 10.1136/bmjopen-2013004706)

Received 18 December 2013 Revised 19 March 2014 Accepted 20 March 2014

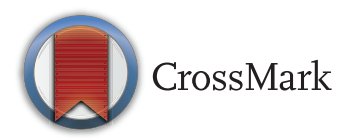

${ }^{1}$ School of Nursing and Midwifery, University of Western Sydney,

Campbelltown Campus, Campbelltown, New South Wales, Australia

${ }^{2}$ School of Medicine, University of Adelaide, Adelaide, South Australia, Australia

${ }^{3}$ School of Science and Health, University of Western Sydney, Campbelltown Campus, Campbelltown, New South Wales, Australia

Correspondence to Dr Evan Atlantis; e.atlantis@uws.edu.au

\section{ABSTRACT}

Objective: The collaborative care model is recommended for depression in adults with a chronic physical health problem like diabetes. We sought to systematically assess the effect of collaborative care on depression and glycaemia in adults with comorbid depression and diabetes to inform guidelines and practice.

Design: Systematic review and meta-analysis. Data sources: We searched PubMed, Scopus, Cochrane Library, CINAHL, Health Source Nursing, MEDLINE, PsychINFO and reference lists of retrieved articles published before August 2013.

Inclusion criteria: Randomised controlled trials (RCTs) on collaborative care (ie, coordinated multidisciplinary model of care) for depression that reported the effects on depression and glycaemic outcomes in adults with comorbid clinically relevant depression and diabetes were eligible.

Data extraction and analysis: Data on the mean difference in depression and glycaemic outcomes were extracted and pooled using random effects meta-analysis.

Results: Seven RCTs included for review reported effects on depression outcomes in 1895 participants, and glycated haemoglobin (HbA1c) level in 1556 participants. Collaborative care significantly improved the depression score (standardised mean difference was $-0.32(95 \% \mathrm{Cl}-0.53$ to -0.11$\left.) ;\left.\right|^{2}=79 \%\right)$ and HbA1c level (weighted mean difference was $-0.33 \%$ $(95 \% \mathrm{Cl}-0.66 \%$ to $\left.-0.00 \%) ;\left.\right|^{2}=72.9 \%\right)$ compared with control conditions. Depression remission did not predict better glycaemic control across studies.

Conclusions: Limited evidence from short-to-medium term RCTs predominantly conducted in the USA suggests that collaborative care for depression significantly improves both depression and glycaemia outcomes, independently, in people with comorbid depression and diabetes.

\section{INTRODUCTION}

Diabetes is currently ranked as the 14th leading cause of global disease burden (assessed using a summary measure of healthy years of life lost due to premature death and years lived with disability), and has

\section{Strengths and limitations of this study}

- The key findings were based on a high-quality systematic review and meta-analysis level of evidence.

- Since only a small number of short-to-medium term studies predominantly conducted in the USA were included, the findings of this review may not be relevant to healthcare settings in other countries, requiring further research.

- Collaborative care for depression significantly improves depression and glycaemia outcomes in people with comorbid depression and diabetes.

moved up several places in the rankings for leading causes since $1990 .{ }^{1}$ The International Diabetes Federation estimated that more than 371 million people (or $8.3 \%$ of the adult population worldwide) had diabetes in $2012 .^{2}$ Major depression, currently ranked the 11th leading cause of global disease burden, has also moved up several places in the rankings for leading causes since $1990 .{ }^{1}$ Although rankings varied substantially across regions, healthcare practitioners in these countries need guidance to better deal with the rising burden of diabetes and depression.

Diabetes is a chronic physical health condition that is often comorbid with clinically relevant symptoms of depression. ${ }^{3-5}$ Practitioners should be aware that depression comorbidity can significantly worsen the self-care, ${ }^{6}$ health $^{7-9}$ and economic burden of diabetes. ${ }^{10}$ This suggests that effective management of depression in people with comorbid diabetes could potentially reverse several of these adverse outcomes, resulting in better glycaemic control among other benefits.

The current National Institute for Health and Care Excellence (NICE) guidelines for depression in adults with a chronic physical health problem, such as diabetes, recommend collaborative care in a 'stepped care framework' in which to organise health services. ${ }^{11}$ Patients with an inadequate response 
to one or more treatments are 'stepped up' from lowintensity care to a more intensive form of management (including lifestyle, psychological and pharmacological therapies). Practitioners should consider collaborative care for patients with comorbid diabetes and depression, since they typically need more intensive care.

Randomised controlled trial (RCT) evidence shows that collaborative care is more effective than usual care for improving depression outcomes both in the short and longer terms in American primary care settings. ${ }^{12}$ Systematic reviews of RCTs have also confirmed that collaborative care is more effective than usual care for improving depression outcomes in people with comorbid diabetes ${ }^{13}{ }^{14}$ but there was a lack of consistent evidence for improving glucose control. ${ }^{13}{ }^{15}$ However, the results of newly published RCTs suggest that collaborative care for depression also leads to significant improvements in glycaemic control. ${ }^{16}{ }^{17}$ We therefore sought to systematically assess the total body of RCT evidence on collaborative care for depression in adults with comorbid depression and diabetes to inform guidelines and practice.

\section{METHODS}

\section{Search strategy}

We searched PubMed, Scopus, Cochrane Library, CINAHL, Health Source Nursing, MEDLINE, PsychINFO and reference lists of retrieved articles published before August 2013. Search syntaxes were developed in consultation with an experienced university research librarian taking into account a broad range of terms and phrases used in definitions of RCTs, collaborative care, depression and diabetes (full electronic search strategies for PubMed and Scopus databases; online supplementary appendix page 1). Reference lists of potentially eligible articles were searched by hand to identify additional studies missed by our search strategy.

\section{Study selection}

Two reviewers (EA and JF) identified potentially relevant studies for inclusion by screening titles and/or abstracts of all citations identified with our database searches. A second screening was performed on the full text of these articles. Articles for RCTs on collaborative care (ie, evidence showing that the intervention was a coordinated multidisciplinary model of care) for depression that reported the effects on both depression and glycaemic outcomes in adults, most of who had to have had comorbid diabetes, were eligible. There were no language restrictions for articles.

\section{Data extraction}

Data extraction and quality assessment of included studies were performed and/or verified independently by three reviewers (EA, JF and PF). Discrepancies were resolved through discussion. Authors of relevant studies were contacted, where possible, for data that could not be extracted from the published articles.

\section{Quality assessment}

For methodology and quality assessment, a quality checklist was developed to identify potential sources of bias (see online supplementary table; appendix page 2). Quality items for RCTs reviewed were as follows (each worth 1.0 numerical point): (1) study eligibility criteria were adequately described, (2) randomisation methodology was adequate (ie, evidence suggesting a 'random' method was used to generate and implement the random allocation sequence), (3) allocation concealment was adequate (ie, evidence to suggest that a robust method was used for concealing the sequence of treatment allocation (eg, independent IT or telephone service or sealed opaque envelopes opened only in front of the participant)), (4) between-group primary outcomes were balanced at baseline (ie, evidence showing that groups were similar at the outset for primary outcomes), (5) between-group dropout rates were balanced and (6) intention to treat analysis was included.

Our quality item checklist was designed based on criteria for assessment of RCTs ${ }^{18} 19$ and allowed summed scores to range from 0 to 6 points, reflecting lowest to highest quality. Studies were considered 'better quality' if they received a score higher than 4 , since that meant that they had most of our quality items.

\section{Primary outcomes}

Data on the mean difference in depression and glycated haemoglobin (HbAlc) outcomes between the treatment and control groups were extracted and pooled using random effects meta-analysis. In one study, ${ }^{16}$ the posttreatment means were derived from the within-group changes and the control group SD carried forward from the baseline values. ${ }^{20}$ Standardised mean differences (SMDs) were calculated using Glass's $\delta$ method.

\section{Data synthesis}

Three reviewers (EA, PF and JF) independently collated and/or verified extracted data to present a descriptive synthesis of important study characteristics and a quantitative synthesis of effect estimates.

\section{Statistical methods}

We pooled and weighted studies first using random effects meta-analysis models, and second using fixed effects models for verification. ${ }^{21}$ Results for HbAlc were pooled to estimate the inverse variance weighted mean difference (WMD), including the DerSimonian and Laird 95\% CI, between treatment and control groups.

In examining the effects of collaborative care treatment on depression scores, the SMD from each RCT was pooled to produce an overall estimate of effect, and associated 95\% CI, between the treatment and control groups. We used meta-regression to test the hypothesis that the SMD in depression score is a predictor of the WMD in HbAlc level.

For each meta-analysis model, the degree of heterogeneity in WMD or SMD was assessed by visual 
inspection, the $\mathrm{I}^{2}$ statistic (moderate being $<50 \%{ }^{22}$ ) and the $\chi^{2}$ test of goodness of fit. ${ }^{23}$ Where evidence of heterogeneity was observed, we checked data extracted from individual outlier studies, qualitatively investigated reasons for their different results and explored the effects of study exclusion in sensitivity analyses.

We also used sensitivity analysis to investigate the robustness of the meta-analyses models. We variously excluded lower quality studies (score of $\leq 4$ ), one study conducted outside the USA (Australia), studies that integrated diabetes care, studies that considered lifestyle risk factors and studies of less than 1-year duration. Publication bias, which reflects the tendency for smaller studies to be published in the literature only when findings are positive, was assessed visually using funnel plots. ${ }^{24}$ All calculations were performed in Stata V.12 (StataCorp, College Station, Texas, USA) using the 'metan', 'metareg' and 'metafunnel' commands. Effects were considered statistically significant when the associated 95\% CIs did not include zero and heterogeneity was considered statistically significant when the associated $p$ value was less than 0.05 .

\section{RESULTS}

Figure 1 presents a flow chart summarising the identification of potentially relevant studies, and those included and excluded. Our search strategy identified 264 citations after duplicates were removed. Of these, 246 citations were excluded after the first screening of titles and/or abstracts for inclusion and exclusion criteria, leaving 18 citations for a second full-text screening. After further assessment, 11 citations were excluded for reasons listed in figure 1, leaving seven RCTs for final inclusion in the systematic review. Most studies were excluded due to inadequate study design or intervention (ie, did not qualify as a collaborative care model), and a couple of studies were excluded for being redundant duplicate citations and for having incomplete data available for extraction (list of excluded citations and reasons; see online supplementary appendix pages 3-4).

\section{Descriptive data synthesis}

Table 1 presents the study characteristics of seven RCTs included for review, which were published between 2004 and 2013. All studies except one ${ }^{25}$ were conducted in the USA. Major inclusion criteria were various case definitions of diabetes in five studies, ${ }^{16} 26-29$ diabetes and/or coronary heart disease in two studies ${ }^{1725}$ and comorbid clinically relevant depression in all studies. Major exclusion criteria were cognitive impairment in four studies, ${ }^{16} 17 \quad 2829$ comorbid psychiatric disorder or suicidal ideation in four studies, ${ }^{17}$ 27-29 alcohol problems in two studies ${ }^{27} 29$ and living in residential care in two studies, ${ }^{17} 25$ among others. The sample sizes ranged from 58 to 417 , resulting in a total of 1895 participants for depression outcomes and 1556 participants for HbA1c outcomes across studies. The mean age of the samples ranged from 54 to 71 years. All the study samples contained both male and female
264 Citations identified from literature search of electronic databases ( 125 duplicates removed)

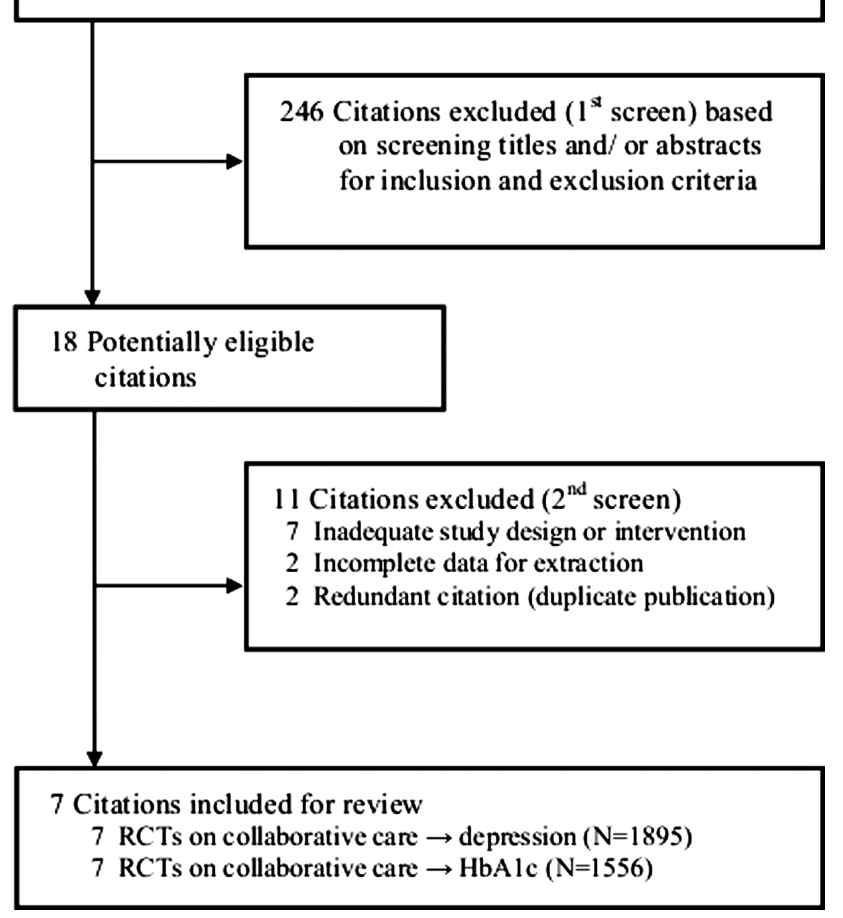

Figure 1 Flow chart summarising identification of studies included for review. HbA1c, glycated haemoglobin; randomised controlled trial (RCT).

participants. Baseline mean depression scores ranged from 15.6 to 19.7 by the CES-D $20,{ }^{26}$ from 9.9 to 11.6 by the Patient Health Questionnaire (PHQ) $-9^{16}{ }^{25}$ and from 1.4 to 1.7 by the Symptom Checklist (SCL)-20. ${ }^{17}$ 27-29 Baseline mean HbA1c levels ranged from $6.9 \%$ to $9.1 \%$. Defining features of collaborative care models investigated were a case manager/officer (usually a nurse or nonphysician mental health worker for coordination of care) with proactive follow-ups in all studies, a structured management plan delivered within a stepped care framework and relapse prevention in four studies, ${ }^{17}$ 27-29 an integrated diabetes care programme in three studies ${ }^{16} 1726$ and consideration for lifestyle risk factors in two studies. ${ }^{17}{ }^{25}$ Control conditions were 'usual care' in four studies, ${ }^{16252629}$ whereas usual care was enhanced in the three other studies. ${ }^{17} 2728$ Trial durations ranged from 12 to 52 weeks. Primary outcomes were depression score assessed by the CES-D 20 in one study, ${ }^{26}$ by the PHQ-9 in two studies ${ }^{1625}$ and by the SCL-20 in four studies ${ }^{17}{ }^{27-29}$; and glycaemic control by HbAlc in all of the studies. Mean quality scores ranged from 3.5 to 5.5 , and all but three studies 252629 received a score of 4.5 or higher.

\section{Quantitative data synthesis}

Effect of collaborative care on depression

Figure 2 presents the SMD in depression outcomes after collaborative care between the treatment and 


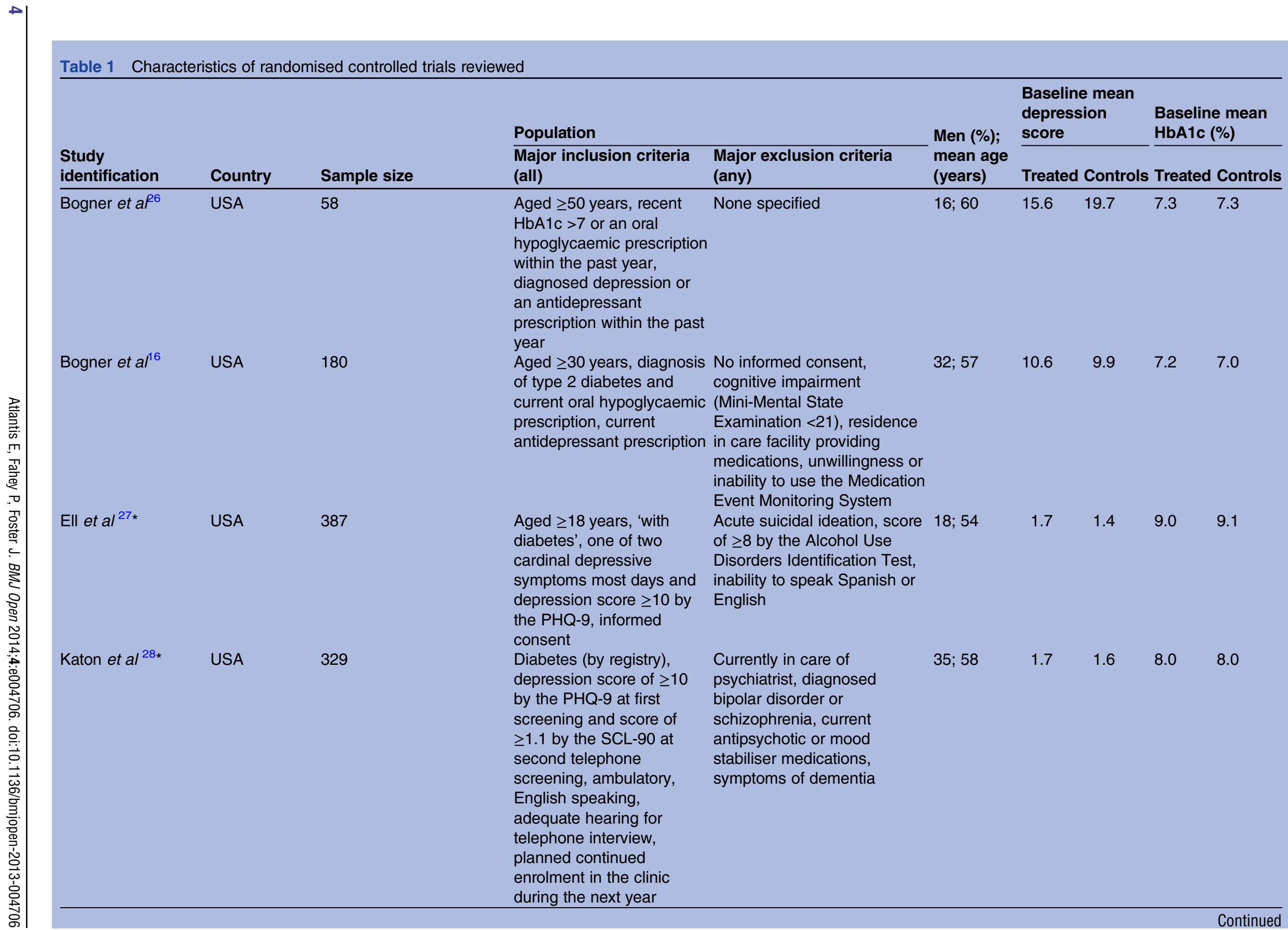




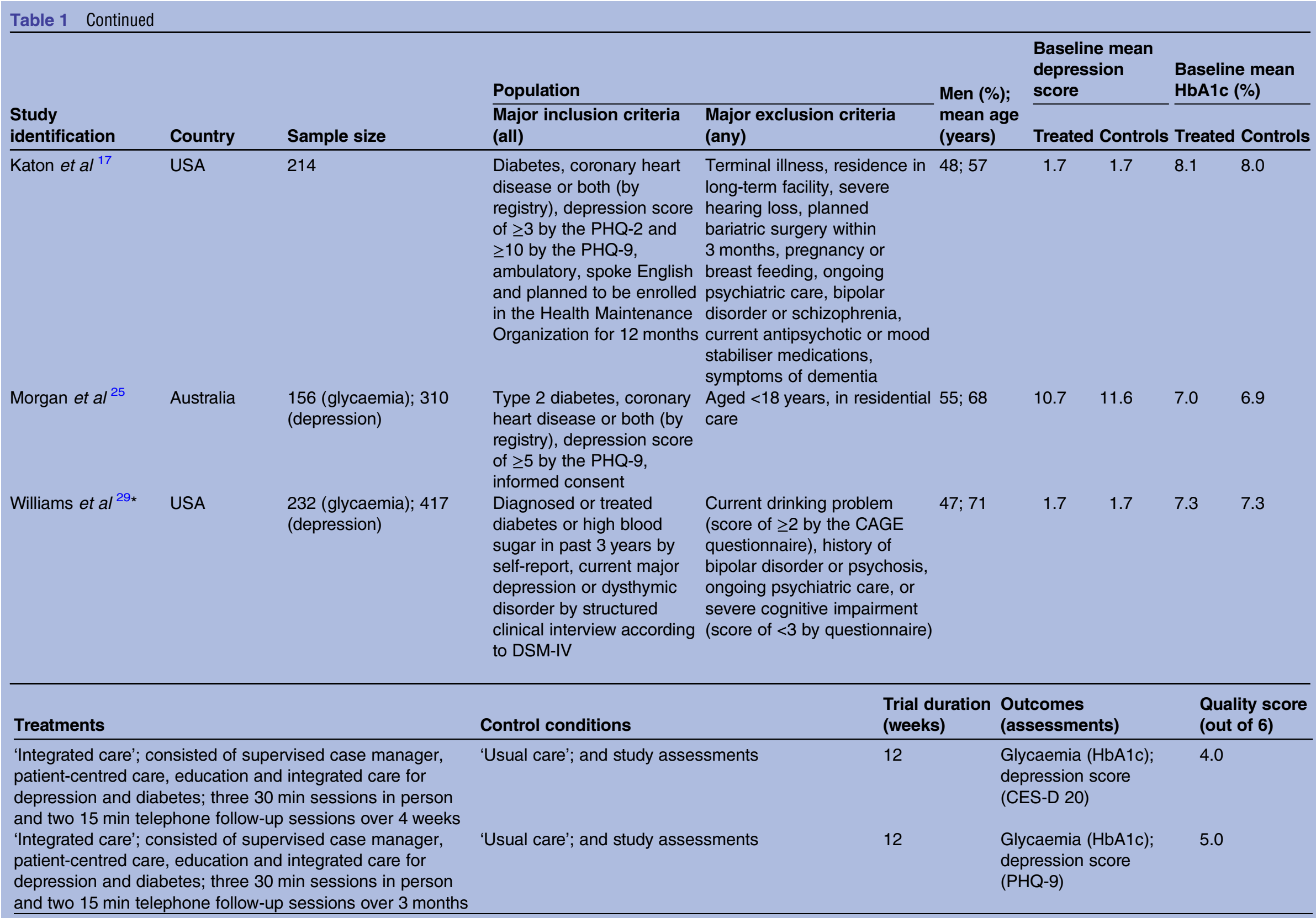




\section{Treatments}

'Collaborative care'; consisted of supervised nurse case manager, patient-centred care, problem solving therapy, self-monitoring education, and coordination of care and services for depression and diabetes within a stepped-care framework; monthly telephone symptom monitoring,

treatment maintenance and relapse prevention up to

12 months

'Collaborative care'; consisted of supervised nurse case manager, patient-centred care (initial choice of

antidepressant or problem solving therapy), within a stepped-care framework; initial one hour visit, followed by

twice monthly half-hour follow-ups (telephone or in-person) up to 12 weeks and referral to specialty care thereafter if necessary

'Collaborative care'; consisted of supervised nurse case manager, 'treat-to-target program' integrated care for specific conditions, within a stepped-care framework, motivational problem solving and goal setting for self-care (including exercise, and 'The Depression Helpbook', video and written material); structured visits every two to 3 weeks, and maintenance plan once targeted levels were achieved including telephone follow-ups every 4 weeks

'Collaborative care'; consisted of collaborative care trained nurse case manager, 45 min nurse consult every 3 months followed (for assessment of lifestyle, physical and

biochemical risk factors, and referrals, self-care of depression and setting personal goals for review and discussion of educational resources), followed by a $15 \mathrm{~min}$ consult with their usual general practitioner

Collaborative care; consisted of trained nurse or psychologist case manager, patient-centred care, problem solving therapy, 20 min educational video tape and written material on late-life depression and coordination of care and services for depression within a stepped-care framework; monthly telephone symptom monitoring, treatment maintenance and relapse prevention up to 12 months;

diabetes care not specifically enhanced

${ }^{*}$ Raw data were provided by the author.

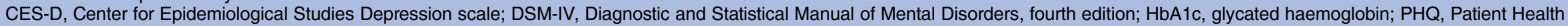
Questionnaire; SCL, Symptom Checklist.
'Usual care'; and advice to consult their physician for depression care

'Enhanced usual care'; patients could self-refer to mental healthcare or be referred by primary care physicians at the clinic and study assessments

'Usual care'; baseline data collected retrospectively
Glycaemia (HbA1c); depression score (PHQ-9)

Quality score (SCL-20)

(SCL-20)
Glycaemia $(\mathrm{HbA} 1 \mathrm{c})$ 
Figure 2 Standardised mean difference (SMD) in depression outcomes after collaborative care between the treatment and control groups.

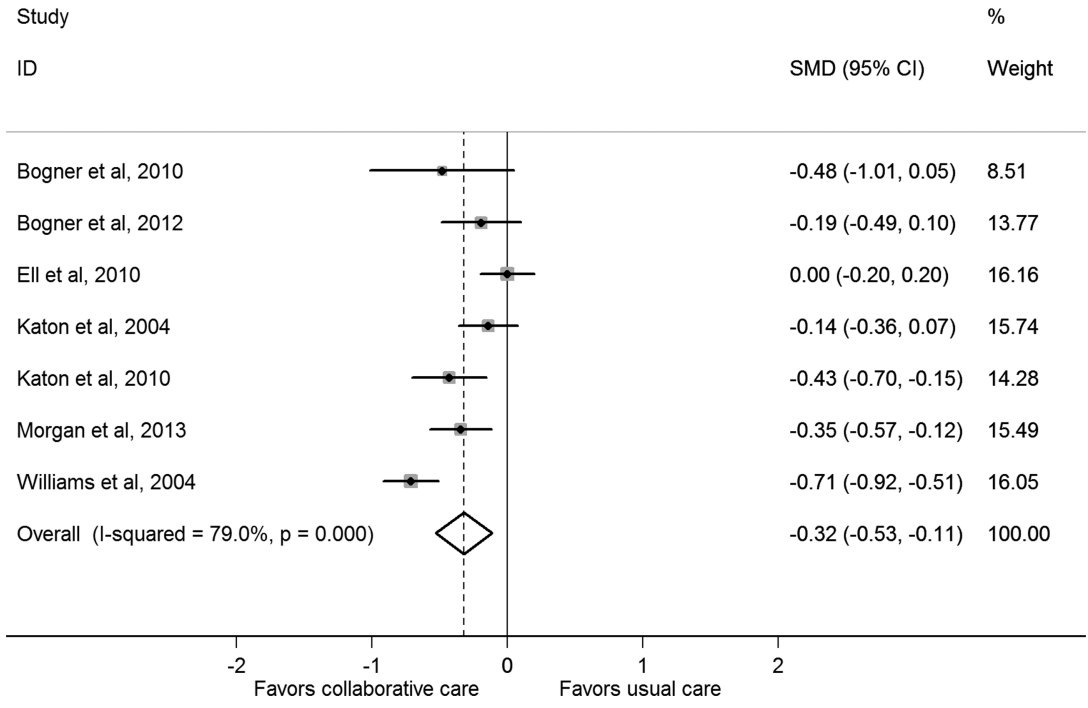

control groups. Collaborative care significantly improved standardised depression outcomes compared with control conditions (pooled SMD was $-0.32(95 \%$ CI -0.53 to -0.11$)$ ). There was statistical heterogeneity between studies $\left(\mathrm{I}^{2}=79 \%, \mathrm{p}<0.001\right)$ that was mostly a result of variation in the degree of benefit favouring collaborative care in all but one study, ${ }^{27}$ which had significant between-group differences in mean depression scores at baseline. Correcting for these differences substantially changed the SMD for that study (from $0.00(95 \%$ CI -0.20 to $0.20)$ to $-0.60(95 \% \mathrm{CI}-0.81$ to -0.39$))$ in a sensitivity analysis. In addition, the sensitivity analyses presented in table 2 show that the pooled SMD was substantially changed only after exclusion of lower quality studies (decreased to -0.17 (95\% CI -0.35 to $0.00)$ ). A funnel plot was produced and confirmed widespread heterogeneity of effect estimates between studies, but did not suggest any publication bias (see online supplementary appendix page 5).

\section{Effect of collaborative care on $\mathrm{HbA1c}$}

Figure 3 presents the WMD in HbAlc level after collaborative care between the treatment and control groups. Collaborative care significantly reduced the HbAlc level compared with control conditions (pooled WMD was $-0.33 \%$ ( $95 \%$ CI $-0.66 \%$ to $-0.00 \%)$ ). There was statistical heterogeneity between studies $\left(\mathrm{I}^{2}=72.9 \%\right.$, $\mathrm{p}=0.001$ ) that was mostly a result of variation in the degree of benefit favouring collaborative care in all but two studies. ${ }^{28}{ }^{29}$ The sensitivity analyses presented in table 3 shows that the pooled WMD was slightly decreased in the fixed effect model $(-0.21$ (95\% CI -0.37 to -0.05$)$ ), but no longer statistically significant after each of the various studies was excluded. This was particularly so after exclusion of three studies that integrated diabetes care (decreased to -0.07 (95\% CI -0.35 to 0.21$)$ ). A funnel plot was produced and confirmed widespread heterogeneity of effect estimates between studies, but did not suggest any publication bias (see online supplementary appendix page 6 ).

\section{Effect of depression remission on $\mathrm{HbA1c}$}

Figure 4 presents a scatter plot displaying the association between the SMD in depression outcomes and the WMD in HbAlc values in each study. Results of a meta-regression model suggest that the SMD for depression scores failed to predict the WMD in HbA1c values across studies ( $\mathrm{p}=0.828$, coefficient was 0.19 (95\% CI -1.93 to 2.31$)$ ).

Table 2 Sensitivity analysis of randomised controlled trials of collaborative care $\rightarrow$ depression score outcome meta-analysis

\begin{tabular}{|c|c|c|c|c|c|}
\hline & $\begin{array}{l}\text { Number of } \\
\text { studies }\end{array}$ & $\begin{array}{l}\text { Number of } \\
\text { sample }\end{array}$ & SMD & $95 \% \mathrm{Cl}$ & $\begin{array}{l}\text { p Value for } \\
\text { heterogeneity }\end{array}$ \\
\hline Fixed effects model & 7 & 1895 & -0.31 & $(-0.40$ to -0.22$)$ & $<0.001$ \\
\hline Exclusion of 3 lower quality studies (score $\leq 4.0$ ) & 4 & 1110 & -0.17 & $(-0.35$ to 0.00$)$ & 0.101 \\
\hline Exclusion of 1 study outside the USA (Australia) & 6 & 1585 & -0.32 & $(-0.57$ to 0.07$)$ & $<0.001$ \\
\hline Exclusion of 3 studies that integrated diabetes care & 4 & 1443 & -0.30 & $(-0.62$ to 0.01$)$ & $<0.001$ \\
\hline $\begin{array}{l}\text { Exclusion of } 2 \text { studies that considered lifestyle risk } \\
\text { factors }\end{array}$ & 5 & 1371 & -0.30 & $(-0.59$ to 0.00$)$ & $<0.001$ \\
\hline Exclusion of 4 studies of less than 1 year duration & 3 & 1018 & -0.34 & $(-0.68$ to 0.01$)$ & $<0.001$ \\
\hline
\end{tabular}


Figure 3 Weighted mean difference (WMD) in glycated haemoglobin level after collaborative care between the treatment and control groups.

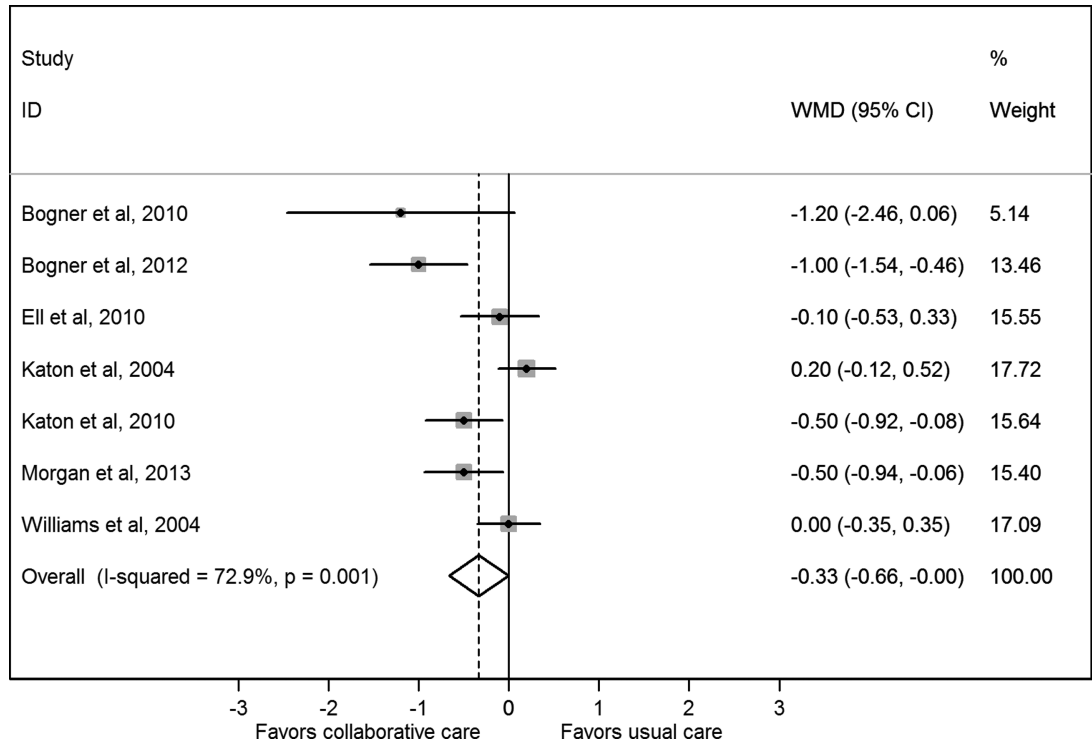

\section{DISCUSSION}

\section{Summary of evidence}

On the basis of the limited evidence from short-to-medium term RCTs predominantly conducted in the USA, our results suggest that collaborative care for depression significantly improves depression and glycaemia outcomes in people with comorbid depression and diabetes. Our results for better glycaemic control are novel and more comprehensive than those published from previous meta-analyses because we sought and obtained raw unpublished data from the authors of three studies. ${ }^{27-29}$ Furthermore, we found evidence from a sensitivity analysis that future high-quality $\mathrm{RCTs}^{30}$ will likely strengthen rather than weaken this evidence base. The size of the effect of collaborative care on depression and HbAlc outcomes that can be expected in practice is small to moderate, but comparable with pharmacological, psychological and behavioural therapies alone, ${ }^{13} 143132$ and likely to be clinically relevant. For instance, several of the RCTs we reviewed have also shown that collaborative care for depression in people with comorbid diabetes is more effective than usual care for improving functional health outcomes ${ }^{33}$ and were cost effective, ${ }^{34}{ }^{35}$ consistent with previous economic modelling. ${ }^{36}$ In addition, a recent meta-analysis found a positive dose-response trend between the HbAlc level and adverse cardiovascular outcomes. ${ }^{37}$ This suggests that improvements in glycaemic control from collaborative care for depression could theoretically protect patients with comorbid diabetes against future cardiovascular risk.

In contrast, we found no evidence to suggest that improved depression outcomes result in better glycaemic control (lower HbAlc values) among people with comorbid diabetes. This null finding for reversibility of the effect of depression on glycaemia weakens the evidence base for causality in terms of worsening the burden of diabetes. Alternatively, collaborative care for depression may improve glycaemia in people with diabetes by increasing self-management, independent of the depression prognosis. For example, collaborative care for depression ${ }^{17}$ was more effective than usual care for improving blood pressure and blood glucose self-monitoring rates. ${ }^{38}$ Quality improvement strategies for diabetes care that promote glucose self-monitoring among patients can significantly improve the HbA1c level (SMD was $0.57 \% \quad(0.31 \%$ to $0.83)) .{ }^{39}$ Indeed, evidence from our sensitivity analysis showed that the effect of collaborative care on HbAlc was

Table 3 Sensitivity analysis of randomised controlled trials of collaborative care $\rightarrow \mathrm{HbA} 1 \mathrm{c}$ outcome meta-analysis

\begin{tabular}{|c|c|c|c|c|c|}
\hline & $\begin{array}{l}\text { Number } \\
\text { of studies }\end{array}$ & $\begin{array}{l}\text { Number } \\
\text { of sample }\end{array}$ & WMD & $95 \% \mathrm{Cl}$ & $\begin{array}{l}\text { p Value for } \\
\text { heterogeneity }\end{array}$ \\
\hline Fixed effects model & 7 & 1556 & -0.21 & $(-0.37$ to -0.05$)$ & 0.001 \\
\hline Exclusion of three lower quality studies (score $\leq 4.0$ ) & 4 & 1110 & -0.32 & $(-0.81$ to 0.17$)$ & 0.001 \\
\hline Exclusion of one study outside the USA (Australia) & 6 & 1400 & -0.31 & $(-0.68$ to 0.07$)$ & 0.001 \\
\hline Exclusion of three studies that integrated diabetes care & 4 & 1104 & -0.07 & $(-0.35$ to 0.21$)$ & 0.086 \\
\hline $\begin{array}{l}\text { Exclusion of two studies that considered lifestyle risk } \\
\text { factors }\end{array}$ & 5 & 1186 & -0.27 & $(-0.71$ to 0.16$)$ & 0.002 \\
\hline Exclusion of four studies of less than 1 year duration & 3 & 833 & -0.18 & $(-0.48$ to 0.11$)$ & 0.189 \\
\hline
\end{tabular}




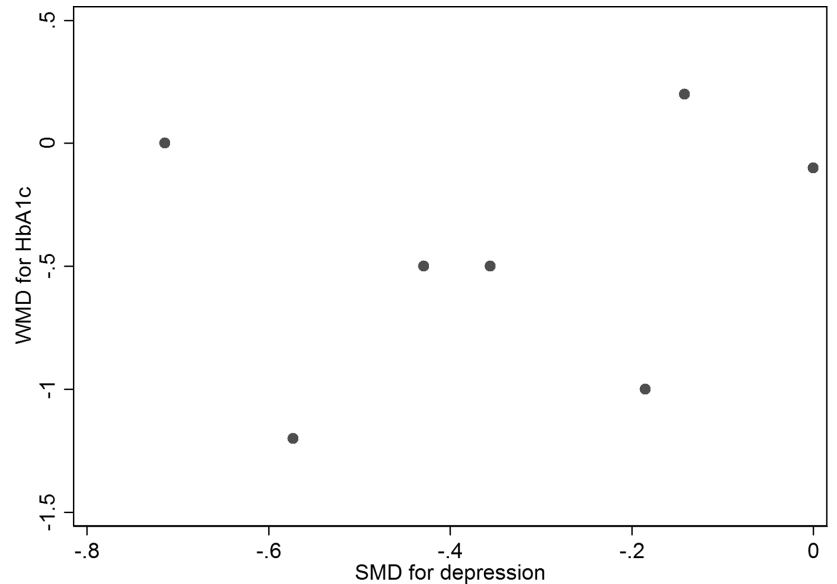

Figure 4 Scatter plot displaying the association between the standardised mean difference (SMD) in depression outcomes and the weighted mean difference (WMD) in glycated haemoglobin values in each study.

almost entirely confined to the three studies that integrated diabetes care within the collaborative care model. ${ }^{161726}$ Second, none of the RCTs that we reviewed properly integrated the lifestyle intervention, as per the current global guideline for effective management of type 2 diabetes, ${ }^{40}$ within the collaborative care. In high-income countries such as the USA and Australia, depression is associated with overweight or obesity, physical inactivity, smoking cigarettes and drinking excessive amounts of sugar-sweetened and alcoholic beverages, ${ }^{41} 42$ all of which are well-established lifestyle risk factors for diabetes. Indeed, there is international consensus supporting the effectiveness of lifestyle intervention in the prevention and management of type 2 diabetes. ${ }^{40}$ In addition, previous systematic reviews of RCTs have shown that exercise (an integral component of lifestyle intervention) is effective for improving the depression score (SMD was $-0.82(95 \%$ CI -1.12 to -0.51$)$ ) and HbA1c level (WMD was $-0.67 \%$ $(95 \% \mathrm{CI}-0.84 \%$ to $-0.49 \%)),{ }^{43} 44$ and the size of these effects is substantially larger than what we found for collaborative care for depression. There is now sufficient evidence to suggest that diabetes care and lifestyle intervention integrated within collaborative care for depression would be the most effective way to manage care for comorbid depression and diabetes.

\section{Limitations}

Several limitations require further consideration. Since only a small number of short-to-medium term studies predominantly conducted in the USA were included, the findings of this review may not be relevant to healthcare settings in other countries, requiring further research. In particular, healthcare systems in most countries are not properly set up to optimise the coordination between practitioners. ${ }^{45}$ Integration of therapies including collaborative care, diabetes care and lifestyle intervention is required to effectively manage comorbid depression diabetes. Second, the baseline mean HbAlc level was close to the upper limit of the normal range in several studies, which would have underestimated the effect size for, and therapeutic benefit of, collaborative care for glycaemic control. Finally, reviewer-level limitations include incomplete retrieval of information for several of the 11 citations excluded, and the existence of other relevant studies not identified with our search strategy resulting in bias. However, the results and conclusions reported in most of the excluded studies were in line with those reported here, and therefore search strategy bias was unlikely.

\section{Conclusions}

The limited evidence from short-to-medium term RCTs predominantly conducted in the USA suggests that collaborative care for depression significantly improves depression and glycaemia outcomes, independently, in people with comorbid depression and diabetes. Future research should investigate the effectiveness, feasibility and appropriateness of collaborative care integration with diabetes care and lifestyle intervention for comorbid depression and diabetes, and other comorbid cardiovascular risk conditions, in routine clinical practice in specific healthcare settings worldwide.

Acknowledgement The authors are grateful to Mrs Rohini Patil for her work on developing and conducting the electronic database searches.

Contributors EA is the guarantor of the paper, taking responsibility for the integrity of the work as a whole, from inception to published article. EA conceived and designed the review, identified studies for inclusion, extracted and interpreted the data and also drafted the article. PF analysed and interpreted the data and revised the article. JF extracted and interpreted the data and revised the article. All authors approved the final completed article.

Funding This research received no specific grant from any funding agency in the public, commercial or not-for-profit sectors.

Competing interests EA has received honoraria for speaking at events for Eli Lilly Australia Pty Ltd (Lilly).

Provenance and peer review Not commissioned; externally peer reviewed.

Data sharing statement No additional data are available.

Open Access This is an Open Access article distributed in accordance with the Creative Commons Attribution Non Commercial (CC BY-NC 3.0) license, which permits others to distribute, remix, adapt, build upon this work noncommercially, and license their derivative works on different terms, provided the original work is properly cited and the use is non-commercial. See: http:// creativecommons.org/licenses/by-nc/3.0/

\section{REFERENCES}

1. Murray CJ, Vos T, Lozano R, et al. Disability-adjusted life years (DALYs) for 291 diseases and injuries in 21 regions, 1990-2010: a systematic analysis for the Global Burden of Disease Study 2010. Lancet 2012;380:2197-223.

2. International Diabetes Federation. IDF diabetes atlas. 5th edn. (C) 2013 International Diabetes Federation, 2013.

3. Atlantis E. Excess burden of type 1 and type 2 diabetes due to psychopathology. J Affect Disord 2012;142(Suppl):S36-41.

4. Lin EHB, Von Korff M, Consortium WWS. Mental disorders among persons with diabetes-results from the World Mental Health Surveys. J Psychosom Res 2008;65:571-80.

5. Moussavi S, Chatterii S, Verdes E, et al. Depression, chronic diseases, and decrements in health: results from the World Health Surveys. Lancet 2007;370:851-8.

6. Lin EHB, Katon W, Von Korff M, et al. Relationship of depression and diabetes self-care, medication adherence, and preventive care. Diabetes Care 2004;27:2154-60.

7. Atlantis E, Goldney RD, Eckert KA, et al. Trends in health-related quality of life and health service use associated with comorbid 
diabetes and major depression in South Australia, 1998-2008. Soc Psychiatry Psychiatr Epidemiol 2012;47:871-7.

8. Lin EHB, Rutter CM, Katon W, et al. Depression and advanced complications of diabetes-a prospective cohort study. Diabetes Care 2010;33:264-9.

9. Park M, Katon WJ, Wolf FM. Depression and risk of mortality in individuals with diabetes: a meta-analysis and systematic review. Gen Hosp Psychiatry 2013;35:217-25.

10. Molosankwe I, Patel A, Gagliardino JJ, et al. Economic aspects of the association between diabetes and depression: a systematic review. J Affect Disord 2012;142:S42-55.

11. National Institute for Health and Clinical Excellence. NICE Clinical Guideline 91. Depression in adults with a chronic physical health problem. London: Treatment and management, 2009.

12. Gilbody S, Bower P, Fletcher J, et al. Collaborative care for depression-a cumulative meta-analysis and review of longer-term outcomes. Arch Intern Med 2006;166:2314-21.

13. Van der Feltz-Cornelis CM, Nuyen J, Stoop C, et al. Effect of interventions for major depressive disorder and significant depressive symptoms in patients with diabetes mellitus: a systematic review and meta-analysis. Gen Hosp Psychiatry 2010;32:380-95.

14. Ekers D, Murphy R, Archer J, et al. Nurse-delivered collaborative care for depression and long-term physical conditions: a systematic review and meta-analysis. J Affect Disord 2013;149:14-22.

15. Huang Y, Wei X, Wu T, et al. Collaborative care for patients with depression and diabetes mellitus: a systematic review and meta-analysis. BMC Psychiatry 2013;13:260.

16. Bogner HR, Morales KH, de Vries HF, et al. Integrated management of type 2 diabetes mellitus and depression treatment to improve medication adherence: a randomized controlled trial. Ann Fam Med 2012;10:15-22.

17. Katon WJ, Lin EH, Von Korff M, et al. Collaborative care for patients with depression and chronic illnesses. N Engl J Med 2010;363:2611-20.

18. CRD's guidance for undertaking reviews in health care. (C) Centre for Reviews and Dissemination University of York 2008Published by CRD. 2009.

19. Schulz KF, Altman DG, Moher D. CONSORT 2010 statement: updated guidelines for reporting parallel group randomised trials. BMJ 2010;340:c332.

20. Higgins JPT, Green S. Cochrane handbook for systematic reviews of interventions version 5.1.0 [updated March 2011]. The Cochrane Collaboration, 2011. http://wwwcochrane-handbookorg [serial on the Internet]. editors.

21. DerSimonian R, Laird N. Meta-analysis in clinical trials. Control Clin Trials 1986;7:177-88.

22. Higgins JP, Thompson SG, Deeks JJ, et al. Measuring inconsistency in meta-analyses. BMJ 2003;327:557-60.

23. Higgins JP, Thompson SG. Quantifying heterogeneity in a meta-analysis. Stat Med 2002;21:1539-58.

24. Egger M, Davey Smith G, Schneider M, et al. Bias in meta-analysis detected by a simple, graphical test. BMJ 1997;315:629-34.

25. Morgan MAJ, Coates MJ, Dunbar JA, et al. The TrueBlue model of collaborative care using practice nurses as case managers for depression alongside diabetes or heart disease: a randomised trial. BMJ Open 2013;3:e002171.

26. Bogner HR, de Vries HF. Integrating type 2 diabetes mellitus and depression treatment among African Americans: a randomized controlled pilot trial. Diabetes Educ 2010;36:284-92.

27. Ell K, Katon W, Xie B, et al. Collaborative care management of major depression among low-income, predominantly hispanic subjects with diabetes: a randomized controlled trial. Diabetes Care 2010;33:706-13.

28. Katon WJ, Korff M, Lin EH, et al. The pathways study: a randomized trial of collaborative care in patients with diabetes and depression.
Arch Gen Psychiatry [serial on the Internet]. 2004;61. http:// onlinelibrary.wiley.com/o/cochrane/clcentral/articles/912/ $\mathrm{CN}-00491912 /$ frame.html

29. Williams JW Jr, Katon W, Lin EHB, et al. The effectiveness of depression care management on diabetes-related outcomes in older patients. Ann Intern Med

2004;140:1015-24+I-51.

30. Johnson JA, Al Sayah F, Wozniak L, et al. Controlled trial of a collaborative primary care team model for patients with diabetes and depression: rationale and design for a comprehensive evaluation. BMC Health Serv Res 2012;12:258.

31. Baumeister H, Hutter N, Bengel J. Psychological and pharmacological interventions for depression in patients with diabetes mellitus and depression. Cochrane Database Syst Rev 2012;12:CD008381.

32. Avery L, Flynn D, van Wersch $A$, et al. Changing physical activity behavior in type 2 diabetes: a systematic review and meta-analysis of behavioral interventions. Diabetes Care 2012;35: 2681-9.

33. Von Korff M, Katon WJ, Lin EH, et al. Functional outcomes of multi-condition collaborative care and successful ageing: results of randomised trial. BMJ (Clin Res ed) 2011;343:d6612.

34. Hay JW, Katon WJ, EII K, et al. Cost-effectiveness analysis of collaborative care management of major depression among low-income, predominantly Hispanics with diabetes. Value Health 2012;15:249-54.

35. Katon W, Russo J, Lin EHB, et al. Cost-effectiveness of a multicondition collaborative care intervention. Arch Gen Psychiatry 2012;69:506-14.

36. Jacob V, Chattopadhyay SK, Sipe TA, et al. Economics of collaborative care for management of depressive disorders: a community guide systematic review. Am J Prev Med 2012;42: $539-49$

37. Zhang Y, Hu G, Yuan Z, et al. Glycosylated hemoglobin in relationship to cardiovascular outcomes and death in patients with type 2 diabetes: a systematic review and meta-analysis. PLOS ONE 2012;7:e42551.

38. Lin EH, Von Korff M, Ciechanowski P, et al. Treatment adjustment and medication adherence for complex patients with diabetes, heart disease, and depression: a randomized controlled trial. Ann Fam Med 2012;10:6-14.

39. Tricco AC, Ivers NM, Grimshaw JM, et al. Effectiveness of quality improvement strategies on the management of diabetes: a systematic review and meta-analysis. Lancet 2012;379: 2252-61.

40. International Diabetes Federation, Clinical Guidelines Task Force. Global guideline for type 2 diabetes. (C) International Diabetes Federation, 2012.

41. Strine TW, Mokdad AH, Dube SR, et al. The association of depression and anxiety with obesity and unhealthy behaviors among community-dwelling US adults. Gen Hosp Psychiatry 2008:30:127-37.

42. Shi Z, Taylor AW, Wittert G, et al. Soft drink consumption and mental health problems among adults in Australia. Public Health Nutr 2010;13:1073-9.

43. Rimer J, Dwan K, Lawlor DA, et al. Exercise for depression Cochrane Database Syst Rev 2012;7:CD004366.

44. Umpierre D, Ribeiro PA, Kramer CK, et al. Physical activity advice only or structured exercise training and association with $\mathrm{HbA} 1 \mathrm{c}$ levels in type 2 diabetes: a systematic review and meta-analysis. JAMA 2011;305:1790-9.

45. Roberts RG, Gask L, Arndt B, et al. Depression and diabetes: the role and impact of models of health care systems. J Affect Disord 2012;142:S80-8. 